\title{
Impact of pelvic bone marrow irradiation on the hematological toxicity of subsequent chemotherapy in rectal cancer
}

\author{
M. SPALEK ${ }^{1, *}$, W. MICHALSKI ${ }^{2}$, L. WYRWICZ ${ }^{2,3}$ \\ ${ }^{1}$ Department of Radiotherapy I, Maria Sklodowska-Curie Institute - Oncology Center, Warsaw, Poland; ${ }^{2}$ Laboratory of Bioinformatics and Bio- \\ statistics, Maria Sklodowska-Curie Institute - Oncology Center, Warsaw, Poland; ${ }^{3}$ Department of Oncology and Radiotherapy, Maria Sklodows- \\ ka-Curie Institute - Oncology Center, Warsaw, Poland
}

${ }^{\star}$ Correspondence: mateusz@spalek.co

Received May 28, 2018 / Accepted June 19, 2018

\begin{abstract}
Preoperative radio(chemo)therapy in rectal cancer may irreversibly damage pelvic bone marrow (PBM) and impair the tolerance of subsequent chemotherapy. The aim of the study was to assess the relationship between the irradiated volume of PBM and the toxicity of subsequent 5-fluorouracil, oxaliplatin, leucovorin (FOLFOX-4) in rectal cancer. We included consecutive rectal cancer patients who received FOLFOX-4 postoperatively or due to cancer relapse. The PBM was divided into iliac (IM), lumbosacral (LSM), and lower pelvic (LPM) marrow. We assessed mean dose, and percentage of volume receiving 10\%-90\% (V10\%-V90\%) of the prescribed dose for PBM, IM, LSM, and LPM. Generalized linear model for repeated measures (GLM) was used to test an influence of dose-volumes distribution on toxicities grade 2 or higher (TOX2) and grade 3 or higher (TOX3). The two-sided t-test was used to evaluate the difference in mean dose, mean V20\%, and mean V40\% between patients who experienced TOX2 or TOX3 and those who did not. 39 patients met eligibility criteria. Because of the low occurrence of TOX3 ( $n=3)$, related analyses were abandoned. We found no influence of dose-volume distribution on TOX2 in GLM and no significant differences in mean dose, mean V20\%, and mean V40\% for PBM, IBM, LSM, and LPM between patients who experienced TOX2 and those who did not. To conclude, no relationship between doses received by $\mathrm{PBM}$ in preoperative radio(chemo)therapy in rectal cancer and hematological tolerance of subsequent FOLFOX-4 chemotherapy was found.
\end{abstract}

Key words: radiation tolerance, adjuvant chemotherapy, chemoradiotherapy, rectal neoplasms

Around half of hematopoietic bone marrow is located in pelvic bones $[1,2]$. All aforementioned bones may be irradiated in case of rectal cancer preoperative radiotherapy. Preoperative radiotherapy may irreversibly damage bone marrow and cause acute or chronic myelosuppression [3]. Hematological toxicity is the main reason of disruptions in chemotherapy administration that may impair the efficacy of systemic treatment. It particularly applies to oxaliplatinbased chemotherapy that often causes myelotoxicity [4]. The question is whether the bone marrow suppression during postoperative oxaliplatin-based chemotherapy that may result from preoperative treatment for rectal cancer is associated with any dose-volume dependence. In the literature there is only one article evaluating this issue [5]. The aim of this retrospective study was to assess the relationship between irradiated volume of pelvic bone marrow and the hematological toxicity of subsequent FOLFOX-4 chemotherapy in rectal cancer.

\section{Patients and methods}

Cohort characteristics. The study is based on pre-defined protocol. A cohort of consecutive patients with adenocarcinoma of rectum who received oxaliplatinbased chemotherapy in 5-fluorouracil, oxaliplatin, leucovorin (FOLFOX-4) regimen postoperatively or because of cancer relapse in our institution between 2011 and 2016 constituted material of this study. The inclusion criteria were: adenocarcinoma, preoperative radiotherapy, radical surgery, and administration of FOLFOX-4 postoperatively or because of the cancer relapse. The exclusion criteria were previous pelvic irradiation, distant metastases at baseline, or missing data in the radiotherapy planning system. FOLFOX-4 was chosen because it is one of the recommended postoperative chemotherapy regimens in rectal cancer. FOLFOX-4 regimen consisted of oxaliplatin administered on day 1 at the dose of $85 \mathrm{mg} / \mathrm{m}^{2}$ in $2 \mathrm{~h}$ infusion, 
concurrently with leucovorin $200 \mathrm{mg} / \mathrm{m}^{2} /$ day, followed by bolus 5 -FU $400 \mathrm{mg} / \mathrm{m}^{2}$ and a $22 \mathrm{~h}$ infusion of 5 -FU $600 \mathrm{mg} /$ $\mathrm{m}^{2}$ for two consecutive days in two week cycles. Because of logistics reasons it was allowed to omit second 5-FU bolus dose and add it to the infusion dose.

Elective clinical target volume (CTV) was contoured according to RTOG Consensus Panel Contouring Atlas [6]. However, our internal protocol allowed for lowering the upper border of CTV from promontory to S2 and S3, depending on localization of the primary tumor or involved lymph nodes. The rationale for adaptation of the local radiotherapy protocol in low-lying tumors was previously described in the literature [7].

Procedures. Varianâ Eclipse Treatment Planning System version 13.6 and Analytical Anisotropic Algorithm version 13.6.23 were used for bone marrow contouring, dose calculation and dose-volume data analysis. Segmentation wizard tool allowed us to contour bone marrow automatically. The pelvic bone marrow (PBM) was divided into three regions: iliac (IM), lumbosacral (LSM), and lower pelvic (LPM) marrow $[1,5]$. IM borders were iliac crest and superior border of the femoral heads. LSM borders were L5 vertebrae and coccyx. LPM included proximal femora, acetabula, pubis, ischia (from the superior border of the femoral heads to the inferior border of the ischial tuberosities).

We assessed mean dose, and percentage of volume receiving gradually $10-90 \%$ (V10-V90\%) of the prescribed dose for PBM, IM, LSM, LPM. Due to the two fractionation schedules, percentage of the prescribed dose was used instead of absolute dose. Mean PBM dose and low doses received by large $\mathrm{PBM}$ volumes have the most important role in the prediction of hematological toxicity $[1,3]$. That gives the rationale to choose mean dose, mean V20\% and mean $\mathrm{V} 40 \%$ as representative parameters in this study.

Hematological toxicities within eight weeks and within 16 weeks from the beginning of chemotherapy were assessed using the Common Terminology Criteria for Adverse Events version 4.03. Toxicities were classified as grade 2 or higher (TOX2) or grade 3 or higher (TOX3). The number of analyzed cycles was pre-specified and was defined accordingly to specific clinical observation. Patients usually received between four and eight cycles because of the planned surgery for synchronous distant metastases. Moreover, 16 weeks of postoperative chemotherapy is recommended for patients after preoperative radiochemotherapy [8]. It gives a rationale for choosing eight and 16 weeks as cut-offs for analysis.

Generalized linear model for repeated measures (GLM) was used to test the influence of dose-volumes (V10-V90\%) distribution on TOX2 and TOX3. The two-sided t-test was used to evaluate the difference in mean dose, mean V20\% and mean V40\% between patients who experienced TOX 2 or TOX3 and those who did not. All $p$-values at level $<0.05$ were considered significant. All analyses were performed using IBM SPSS Statistics version 23.

\section{Results}

Thirty-nine patients met eligibility criteria. 26 patients were irradiated with $5 \times 5$ Gy regimen alone, seven received $5 \times 5$ Gy and three cycles of consolidation FOLFOX-4, and six underwent conventionally fractionated radiochemotherapy (25×2 Gy + concomitant bolus 5-FU and leucovorin). In all analyzed patients, three dimensional conformal radiation therapies (3D-CRT) were used. In $64 \%(n=25)$ of patients the upper clinical target volume border was set at S2/S3 interface and in the remaining $36 \%(\mathrm{n}=14)$ at promontory. Patients' characteristics are shown in Table 1 . The median time from the end of radiotherapy and the first dose of FOLFOX-4 was 79 days (interquartile range [IQR]: 111).

TOX2 occurred in $38 \%(n=15)$ of patients. TOX3 occurred only in three patients. Due to low power, the planned statistical analyses related to TOX3 were abandoned. We found no influence of dose-volume distribution on TOX2 in GLM (Figure 1). We did not find significant differences in mean dose, mean V20\% and mean V40\% for PBM, IBM, LSM, and LPM between patients who experienced TOX2 and those who did not. Results are shown in Table 2.

\section{Discussion}

In this study, the dosimetric parameters for preoperative radio(chemo)therapy in patients with rectal cancer were not associated with the toxicity of subsequent chemotherapy in FOLFOX-4 regimen. This study is the second attempt to assess the long-term impact of pelvic bone marrow irradiation on the hematological toxicity of subsequent chemotherapy. Newman et al. examined bone marrow suppression during postoperative FOLFOX in a group of rectal cancer patients $(n=35)$ who underwent preoperative radiochemotherapy [5]. To establish the association of dose-volume parameters for each bone marrow area with grade $3+$ toxicities and the occurrence of hematological events, authors used univariate and multivariate analyses. Then receiver operator curves were calculated for significant dose-volume parameters on

Table 1. Patient characteristics.

\begin{tabular}{lc}
\hline & $\begin{array}{c}\text { Analyzed group } \\
\mathbf{n}=\mathbf{3 9}\end{array}$ \\
\hline $\begin{array}{l}\text { Median age in years } \\
\text { (interquartile range) }\end{array}$ & 63 \\
Sex: & $(55-69)$ \\
$\quad$ Male & \\
Female & $29(74.4 \%)$ \\
FOLFOX-4 indication & $10(25.6 \%)$ \\
Adjuvant & \\
Palliative & $27(69.2 \%)$ \\
Prior to resection of limited metastases & $1(2.6 \%)$ \\
\hline
\end{tabular}

* at the moment of first FOLFOX-4 administration;

Abbreviations: FOLFOX-4 - 5-fluorouracil, oxaliplatin, leucovorin 
A
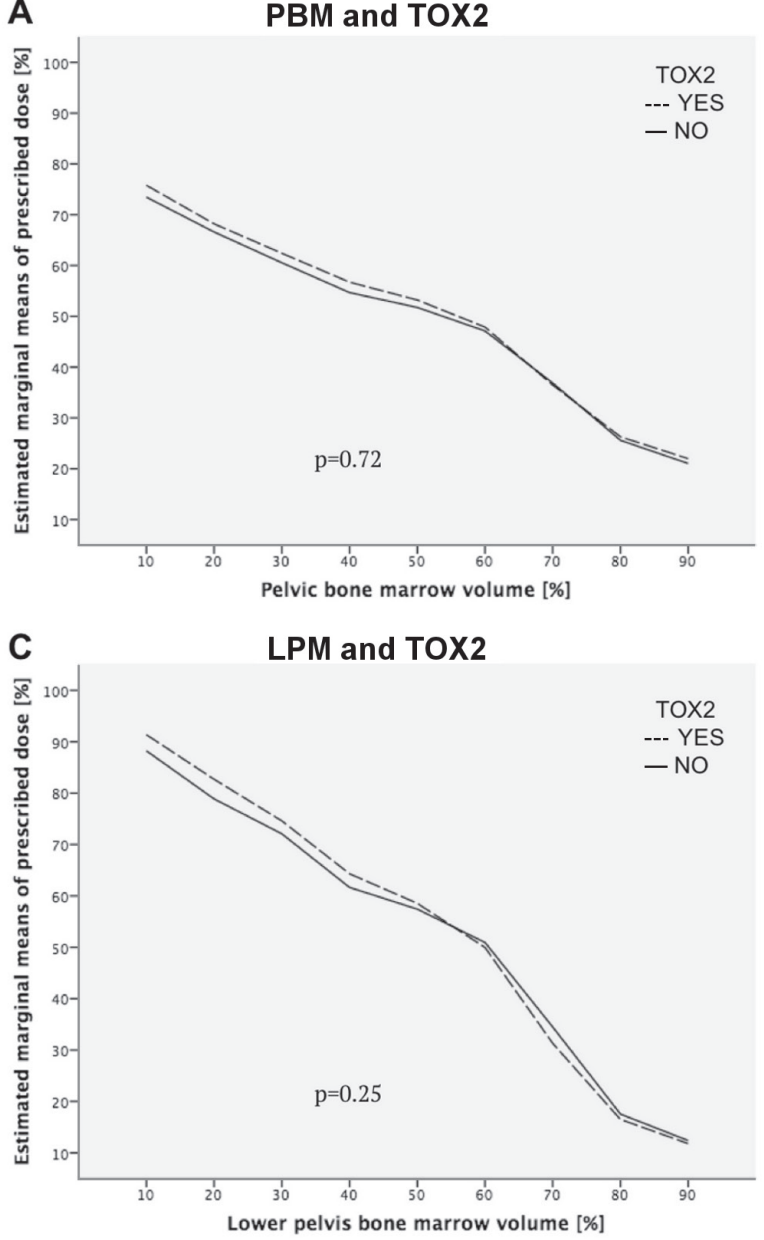

B

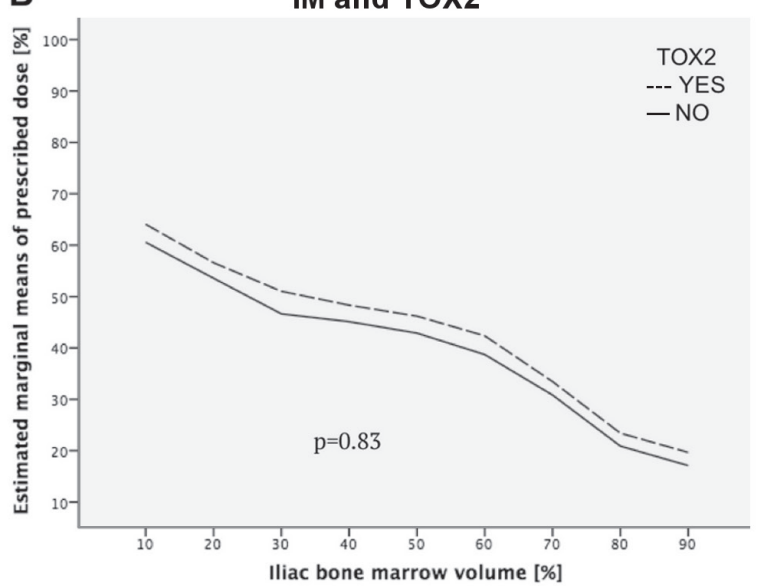

D

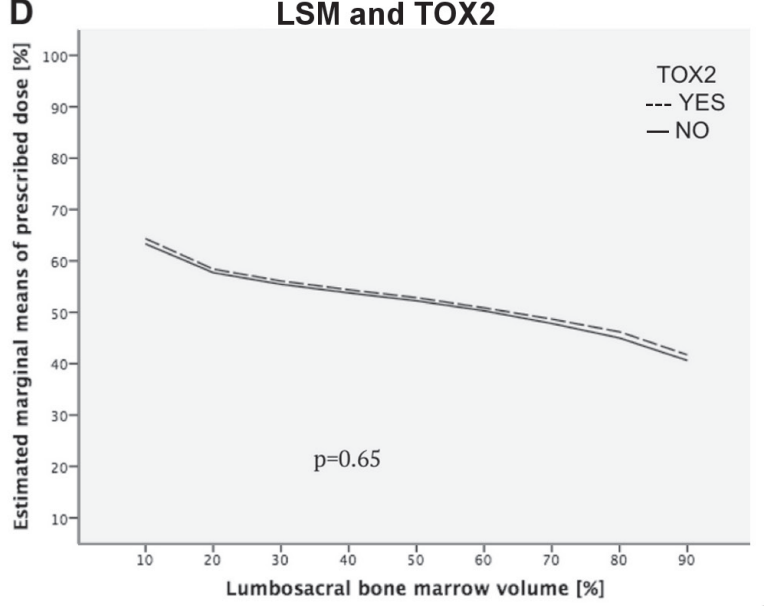

Figure 1. Generalized linear model for repeated measures for an influence of dose-volumes distribution on hematological toxicity. Generalized linear model for repeated measures for influence of preoperative radiotherapy dose received by bone marrow volume and its sub-volumes on grade 2 or higher hematological toxicity (TOX2). The four figures present dose-volume distributions and their relation to TOX2 for pelvic bone marrow (A), iliac bone marrow (B), lower pelvis bone marrow (C), lumbosacral bone marrow (D). An influence of dose-volume distribution on TOX2 was not found in any analyzed bone marrow volumes. Abbreviations: IM - iliac bone marrow; LPM - lower pelvis bone marrow; LSM - lumbosacral bone marrow; PBM - pelvic bone marrow; TOX2 - hematological toxicity grade 2 or higher.

Table 2. Bone marrow dose-volume effect on hematological toxicity.

\begin{tabular}{|c|c|c|c|c|c|}
\hline \multirow[b]{2}{*}{ Area of BM } & \multirow{2}{*}{\multicolumn{2}{|c|}{ Mean volume $(\mathrm{SD})\left[\mathrm{cm}^{3}\right]$}} & \multicolumn{2}{|c|}{$\operatorname{Mean}^{\star}(\mathrm{SD})[\%]$} & \multirow[b]{2}{*}{ p-value } \\
\hline & & & $\begin{array}{l}\text { TOX2 } \\
n=15\end{array}$ & $\begin{array}{c}\text { No TOX2 } \\
n=24\end{array}$ & \\
\hline \multirow{3}{*}{ Pelvic } & \multirow{3}{*}{$\begin{array}{c}1600.1 \\
( \pm 287.3)\end{array}$} & mean dose & $50.5( \pm 9.0)$ & $48.9( \pm 9.8)$ & 0.61 \\
\hline & & V20\% & $68.2( \pm 11.0)$ & $66.6( \pm 12.6)$ & 0.69 \\
\hline & & V $40 \%$ & $56.7( \pm 12.9)$ & $54.8( \pm 13.1)$ & 0.64 \\
\hline \multirow{3}{*}{ Iliac } & \multirow{3}{*}{$\begin{array}{c}496.7 \\
( \pm 94.7)\end{array}$} & mean dose & $43.9( \pm 11.0)$ & $39.9( \pm 14.1)$ & 0.36 \\
\hline & & V20\% & $56.6( \pm 14.9)$ & $53.6( \pm 19.6)$ & 0.61 \\
\hline & & $\mathrm{V} 40 \%$ & $48.3( \pm 14.7)$ & $45.1( \pm 18.3)$ & 0.57 \\
\hline \multirow{3}{*}{ Lumbosacral } & \multirow{3}{*}{$\begin{array}{c}402.4 \\
( \pm 67.8)\end{array}$} & mean dose & $53.8( \pm 17.4)$ & $51.9( \pm 19.9)$ & 0.76 \\
\hline & & V20\% & $58.4( \pm 19.2)$ & $57.7( \pm 22.4)$ & 0.92 \\
\hline & & $\mathrm{V} 40 \%$ & $54.4( \pm 18.9)$ & $53.8( \pm 22.3)$ & 0.93 \\
\hline \multirow{3}{*}{ Lower pelvic } & \multirow{3}{*}{$\begin{array}{c}693.2 \\
( \pm 137.6)\end{array}$} & mean dose & $53.4( \pm 14.3)$ & $53.4( \pm 18.5)$ & 1.00 \\
\hline & & V20\% & $82.7( \pm 14.3)$ & $78.9( \pm 18.5)$ & 0.50 \\
\hline & & V $40 \%$ & $64.3( \pm 18.6)$ & $61.7( \pm 16.1)$ & 0.64 \\
\hline
\end{tabular}

* mean dose as percentage of prescribed dose; Abbreviations: BM - bone marrow; SD - standard deviation; TOX2 - hematological toxicity grade 2 or higher 
logistic regression. Authors found that increasing pelvic bone marrow dose-volume parameters were significantly associated with the occurrence of grade $3+$ hematological toxicity during postoperative chemotherapy.

The difference between the above mentioned results and those found in our study may be caused by different radiotherapy techniques and target size. In the aforementioned analysis, majority of patients $(57.1 \%)$ were treated with intensity-modulated radiation therapy (IMRT), whereas all our patients received 3D-CRT. They included patients treated according to RTOG 0882 protocol in which IMRT planning was focused on intestinal rather than marrow sparring. Exposure of bone marrow to low doses is significantly higher for IMRT than in 3D-CRT technique $[9,10]$. Thus larger volume of healthy marrow is affected by radiation by IMRT than by 3D-CRT. Moreover, majority of our patients (64\%) had the upper clinical target volume border set at S2/S3, whereas all patients from the aforementioned study had the upper border set at promontory. This may decrease the volume of irradiated LSM, and translate into better treatment tolerance. The authors of above-mentioned study included patients who underwent conventionally fractionated radiochemotherapy, whereas majority of our patients received short-course radiotherapy. Clinical data regarding effects of short-course radiotherapy on hematological toxicity of subsequent chemotherapy is lacking in the literature. However, biologically effective dose according to the time-corrected linear quadratic model for late effects $(\alpha /$ $\beta=3 \mathrm{~Gy}$ ) shows that late toxicity is at least not higher after hypofractionated radiotherapy than after conventionally fractionated radiotherapy (66.7 Gy in $5 \times 5$ Gy vs $72-84$ Gy in 45-50.4 Gy in 1.8-2.0 Gy fractions) [11]. The impact of administration and sequence of preoperative chemotherapy may be also important. In our study majority of patients did not receive preoperative chemotherapy. Among these who received preoperative systemic treatment, six patients underwent conventionally fractionated radiochemotherapy without oxaliplatin, whereas in seven patients $5 \times 5$ Gy radiotherapy with consolidative 3 courses of FOLFOX-4 was given. In the Polish phase III randomized study, authors compared acute hematological toxicity between these two regimens [12]. It was lower in the $5 \times 5$ Gy with consolidation FOLFOX-4 group than in the concomitant radiochemotherapy group. In our study, the size of two groups with chemotherapy is too small to detect any difference. Moreover, the time period between the end of radiotherapy and the first dose of subsequent chemotherapy may play important role in predicting hematological toxicity. Longer interval for recovery may result in better chemotherapy tolerance. Newman et al. included only patients treated postoperatively, whereas we also included patients who received FOLFOX-4 prior to resection of limited metastases (28.2\%) and in palliative setting (2.6\%) [5]. Nevertheless, the median time between the end of preoperative treatment and subsequent chemotherapy was comparable between our study and above-mentioned study, 79 days (IQR: 111) and 105 days (IQR: 52), respectively.

High doses received by large volumes of bone marrow could irreversibly damage bone marrow hematopoietic cells. However, adult human blood also contains progenitor cells that are able to recover marrow hematopoiesis [13]. A murine model showed that hypofractionated radiotherapy resulted in a pronounced, targeted recruitment and colony formation of bone-marrow-derived hematopoietic stem and progenitor cells [14]. This mechanism may be related to the phenomenon called bone marrow reconversion, where inactive yellow bone marrow is replaced with active red bone marrow [15]. Irradiation of $50 \%$ or more of the bone marrow volume causes intensive attempts to compensate for the myelosuppression [13]. The reconversion phenomenon then begins in non-irradiated healthy bones. In-field marrow regeneration was also described; usually several years after irradiation.

This study has limitations. A retrospective nature of the analysis may have introduced selection bias. Small sample size could lead to type II error (false negative results). In conclusion, we failed to find any association between dose received by $\mathrm{PBM}$ in preoperative radio(chemo)therapy in rectal cancer and hematological toxicity of subsequent FOLFOX-4.

Acknowledgements: We thank Sebastian Rybski MSc for assistance with electronical medical and data extraction.

\section{References}

[1] MELL LK, KOCHANSKI JD, ROESKE JC, HASLAM JJ, MEHTA N et al. Dosimetric predictors of acute hematologic toxicity in cervical cancer patients treated with concurrent cisplatin and intensity-modulated pelvic radiotherapy. Int J Radiat Oncol Biol Phys 2006; 66: 1356-1365. https://doi. org/10.1016/j.ijrobp.2006.03.018

[2] HAYMAN JA, CALLAHAN JW, HERSCHTAL A, EVERITT $S$, BINNS DS et al. Distribution of proliferating bone marrow in adult cancer patients determined using FLT-PET imaging. Int J Radiat Oncol Biol Phys 2011; 79: 847-852. https://doi. org/10.1016/j.ijrobp.2009.11.040

[3] MELL LK, SCHOMAS DA, SALAMA JK, DEVISETTY $\mathrm{K}$, AYDOGAN B et al. Association between bone marrow dosimetric parameters and acute hematologic toxicity in anal cancer patients treated with concurrent chemotherapy and intensity-modulated radiotherapy. Int J Radiat Oncol Biol Phys 2008; 70: 1431-1437. https://doi.org/10.1016/j. ijrobp.2007.08.074

[4] TOURNIGAND C, ANDRÉ T, ACHILLE E, LLEDO G, FLESH $\mathrm{M}$ et al. FOLFIRI followed by FOLFOX6 or the reverse sequence in advanced colorectal cancer: a randomized GERCOR study. J Clin Oncol 2004; 22: 229-237. https://doi. org/10.1200/JCO.2004.05.113 
[5] NEWMAN NB, SIDHU MK, BABY R, MOSS RA, NISSENBLATT MJ et al. Long-Term Bone Marrow Suppression During Postoperative Chemotherapy in Rectal Cancer Patients After Preoperative Chemoradiation Therapy. Int J Radiat Oncol Biol Phys 2016; 94: 1052-1060. https://doi. org/10.1016/j.ijrobp.2015.12.374

[6] MYERSON RJ, GAROFALO MC, EL NAQA I, ABRAMS RA, APTE A et al. Elective clinical target volumes for conformal therapy in anorectal cancer: a radiation therapy oncology group consensus panel contouring atlas. Int J Radiat Oncol Biol Phys 2009; 74: 824-830. https://doi.org/10.1016/j. ijrobp.2008.08.070

[7] NIJKAMP J, KUSTERS M, BEETS-TAN RGH, MARTIJN H, BEETS GL et al. Three-dimensional analysis of recurrence patterns in rectal cancer: the cranial border in hypofractionated preoperative radiotherapy can be lowered. Int J Radiat Oncol Biol Phys 2011; 80: 103-110. https://doi.org/10.1016/j. ijrobp.2010.01.046

[8] WILLETT CG. Management of Locoregional Rectal Cancer. J Natl Compr Canc Netw 2018; 16: 617-619. https://doi. org/10.6004/jnccn.2018.0037

[9] YOO S, WU QJ, LEE WR, YIN FF. Radiotherapy treatment plans with RapidArc for prostate cancer involving seminal vesicles and lymph nodes. Int J Radiat Oncol Biol Phys 2010; 76: 935-942. https://doi.org/10.1016/j.ijrobp.2009.07.1677
[10] BINDHU J, SUPE S, PAWAR Y. Intensity modulated radiotherapy (IMRT) the white, black and grey: a clinical perspective. Rep Pract Oncol Radiother 2009; 14: 95-103. https:// doi.org/10.1016/S1507-1367(10)60101-0

[11] GLIMELIUS B. Neo-adjuvant radiotherapy in rectal cancer. World J Gastroenterol 2013; 19: 8489-8501. https://doi. org/10.3748/wjg.v19.i46.8489

[12] BUJKO K, WYRWICZ L, RUTKOWSKI A, MALINOWSKA $\mathrm{M}$, PIETRZAK L et al. Long-course oxaliplatin-based preoperative chemoradiation versus 5 x 5 Gy and consolidation chemotherapy for cT4 or fixed cT3 rectal cancer: results of a randomized phase III study. Ann Oncol 2016; 27: 834-842. https://doi.org/10.1093/annonc/mdw062

[13] MAUCH P, CONSTINE L, GREENBERGER J, KNOSPE W, SULLIVAN J et al. Hematopoietic stem cell compartment: acute and late effects of radiation therapy and chemotherapy. Int J Radiat Oncol Biol Phys 1995; 31: 1319-1339. https:// doi.org/10.1016/0360-3016(94)00430-S

[14] KANE J, KRUEGER SA, DILWORTH JT, TORMA JT, WILSON GD et al. Hematopoietic stem and progenitor cell migration after hypofractionated radiation therapy in a murine model. Int J Radiat Oncol Biol Phys 2013; 87: 1162-1170. https://doi.org/10.1016/j.ijrobp.2013.08.044

[15] MALKIEWICZ A, DZIEDZIC M. Bone marrow reconversion - imaging of physiological changes in bone marrow. Pol J Radiol 2012; 77: 45-50. 